\title{
Policy-Enhanced Private Set Intersection: Sharing Information While Enforcing Privacy Policies
}

\author{
Emil Stefanov, Elaine Shi, and Dawn Song \\ UC Berkeley \\ \{emil, elaines, dawnsong\}@cs. berkeley.edu
}

\begin{abstract}
Companies, organizations, and individuals often wish to share information to realize valuable social and economic goals. Unfortunately, privacy concerns often stand in the way of such information sharing and exchange.

This paper proposes a novel cryptographic paradigm called PolicyEnhanced Private Set Intersection (PPSI), allowing two parties to share information while enforcing the desired privacy policies. Our constructions require minimal additional overhead over traditional Private Set Intersection (PSI) protocols, and yet we can handle rich policy semantics previously not possible with traditional PSI and Authorized Private Set Intersection (APSI) protocols. Our scheme involves running a standard PSI protocol over carefully crafted encodings of elements formed as part of a challenge-response mechanism. The structure of these encodings resemble techniques used for aggregating BLS signatures in bilinear groups. We prove that our scheme is secure in the malicious model, under the CBDH assumption, the random oracle model, and the assumption that the underlying PSI protocol is secure against malicious adversaries.
\end{abstract}

\section{Introduction}

The need for two parties to exchange privacy-sensitive information arises in numerous application domains. Often, the two parties involved in the exchange are mutually distrustful and do not wish to reveal any additional information other than what is necessary. In particular, we consider the scenario where two parties each hold a set of elements and wish to find the intersection of their elements without revealing other elements that are not in the intersection. In such applications, it is important to ensure that each data item being exchanged

\footnotetext{
* This material is based upon work partially supported by the Air Force Office of Scientific Research under MURI Grant No. 22178970-4170 and No. FA9550-08-1-0352, by the National Science Foundation Graduate Research Fellowship under Grant No. DGE-0946797, by Intel through the ISTC for Secure Computing, and by a grant from the Amazon Web Services in Education program. Any opinions, findings, and conclusions or recommendations expressed in this material are those of the author(s) and do not necessarily reflect the views of the funding agencies.
} 
is properly authenticated or authorized by the owner(s) or creator(s) of that data item due to the following reasons:

- Thwart dishonest behavior. Unless some form of authentication is required, a malicious party can claim possession of fictitious data items, in an attempt to find out whether the other party possesses these data items. For example, if hospitals $A$ and $B$ are trying to find out their common patients, a malicious hospital $A$ can fictitiously claim that Carol is their patient, in an attempt to find out whether Carol is a patient with hospital $B$.

- Comply with privacy policies. Sharing of privacy-sensitive information may be governed by certain privacy regulations, either made by the government or individual organizations. For example, two healthcare providers $A$ and $B$ may wish to exchange information about their common patients to improve service and facilitate diagnosis. However, due to privacy regulations such as the Health Insurance Portability and Accountability Act (HIPAA), they can only share a patient's record if both providers have obtained the patient's consent. The above is an example of a simple privacy policy. In other application scenarios, we may also desire the ability to support richer privacy policies. We demonstrate how to support rich privacy policies in Section 4

In this paper, we propose Policy-Enhanced Private Set Intersection (PPSI). In PPSI, each party has a set of elements, where each element may be authorized (signed) by a different authority or authorities. PPSI allows two parties to find the intersection of their sets, while enforcing rich privacy policies. The policies specify what authorizations each party must possess for its elements. Our scheme thwarts dishonest behavior by preventing a malicious party from using unauthorized elements during the set intersection to violate the privacy of the other party.

\subsection{Results and Contributions}

New problem definitions One important contribution we make is the definition of a new problem, namely, Policy-Enhanced Private Set Intersection (PPSI). Existing Private Set Intersection (PSI) protocols and Authorized Private Set Intersection (APSI) protocols are not general enough and fail to adequately address the needs of above-mentioned application scenarios. To resolve this problem our PPSI scheme offers the following rich capabilities not previously possible with existing PSI and APSI protocols:

- Multiple authorities. PPSI supports privacy policies where each element may be authorized by a different authority or different authorities. This makes PPSI particularly useful when each data item may not be owned or created by the same entity.

- Rich privacy policies. Many applications desire the ability to support expressive privacy policies. Our PPSI constructions can support rich policy 
Table 1. Efficiency of our construction in Section 3.3. $n$ is the maximum number of elements per user and $m$ is the maximum number of authorities per element. The complexities are calculated assuming that we use [15] as the underlying PSI scheme.

\begin{tabular}{|c|c|c|}
\hline & Overall & Additional overhead over PSI \\
\hline Computation & $O(n m+n \log \log n)$ & at most $n m$ pairings \\
\hline Bandwidth & $O(n)$ & 2 group elements \\
\hline \# Rounds & $O(1)$ & 1 round \\
\hline
\end{tabular}

semantics during the information sharing process, including conjunctive and disjunctive policies, asymmetric policies, policies with attributes, and bundles of elements.

Novel, Provably Secure Constructions. We propose novel PPSI constructions that offer two main functionalities: 1) a signing functionality which allows an authority to authenticate or authorize an element for a party; 2) a set intersection protocol that allows two parties to find the intersection of their elements, while enforcing the desired privacy policy.

We prove the security of our scheme against malicious adversaries, assuming that the underlying PSI scheme is also secure in the malicious model. The proof also relies on the $\mathrm{CBDH}$ assumption and the random oracle model.

Efficiency. Our constructions are efficient in practice. Specifically, we require $O(n)$ communication bandwidth and $O(n m+n \log \log n)$ computation, where $n$ is the maximum number of elements per party and $m$ is the maximum number of authorities per element. Also, our protocol executes in $O(1)$ rounds.

Notably, our constructions require only minimal overhead over standard Private Set Intersection (PSI) protocols, but can support rich policies that are not possible with standard PSI. We need one additional round of communication over standard PSI, during which the parties exchange two group elements (elliptic points). In terms of computation, we incur an additional overhead of at most $\mathrm{nm}$ bilinear pairings over the traditional PSI protocols.

Table 1 summarizes the efficiency of our basic construction described in Section 3.3 .

\subsection{Technical Challenges and Highlight of Our Techniques}

It turns out that the problem is non-trivial, even with relatively simple policies. A straightforward idea is to adopt an existing PSI protocol and require that each party demonstrate a zero-knowledge proof that each element encoded in a cryptographic commitment has the appropriate authorizations. However, the complication is that when each element has a different authority or different authorities, one cannot reveal the identity of the authority when performing the zero-knowledge proofs, as the identity of the authority can leak information about the corresponding element. 
Special encodings. Our techniques may be of independent interest. Our scheme leverages a type of special encoding that allows us to circumvent the need for performing complicated and costly zero-knowledge proofs. Each party computes the special encodings over their elements and then runs a standard PSI protocol over these encodings.

A party's encoding for an element $x$ is essentially a product of terms demonstrating its authorization on $x$ and the anticipated terms demonstrating the other party's authorization on $x$. The terms are cleverly crafted so that a party can compute its own terms by combining its own authorizations and a challenge sent by the other party. It can also compute the anticipated terms of the other party without having the other party's authorizations.

If both parties satisfy their respective policies for an element, then both parties obtain the same encoding for that element, and this particular encoding appears in the set intersection. However, if a party does not possess the correct authorizations for an element, it is unable (computationally intractable) to compute the correct encoding for this element. As a result, this party is unable to learn whether the other party owns the element.

We point out that our encoding idea bears resemblance to techniques used for aggregating BLS signatures in bilinear groups [5].

\subsection{Related Work}

A Private Set Intersection (PSI) protocol [9, 10, 11, 12, 13, 14, 15, 16, 17, 18. allows two parties to find the intersection of their respective sets such that neither party can infer elements in the other party's set that are not in the intersection. However, PSI protocols allow each party to place any element in their own set. A dishonest party can therefore insert fabricated elements in its set that she suspects the other party might have. The intersection will reveal if the other party indeed has those elements in its set.

To address this issue, Authorized Private Set Intersection (APSI) and variants [6, 8, 10] ensure that each party can only use elements certified by a trusted authority in the intersection protocol. Existing APSI protocols assume that for each party, there exists a single authority responsible for certifying all of its elements. Therefore, these schemes do not support rich privacy policies coming from multiple authorities, such as the application scenarios mentioned earlier.

\section{Problem Definitions}

\subsection{Notations and Terminology}

Let $\mathcal{U}$ denote a (countable) universe of all possible elements. Let $\Lambda$ denote the set of all authorities.

As mentioned earlier, two parties, $P_{A}$ and $P_{B}$, wish to find the intersection of their sets in a way that complies with certain privacy policies, that is, only when both parties have the appropriate authorizations for an element should it appear in the intersection. 
Table 2. Table of notations

\begin{tabular}{|c|c|}
\hline$n$ & Max number of elements in each party's set. \\
\hline$m$ & Max number of authorities per element. \\
\hline $\mathcal{U}$ & The set of all possible elements. \\
\hline$x \in \mathcal{U}$ & An element. \\
\hline$\Lambda$ & The set of all authorities \\
\hline auth $_{i} \in \Lambda$ & An authority that signs elements. \\
\hline$P_{i}$ & A party participating in our protocol. \\
\hline$S_{i}$ & $P_{i}$ 's set. \\
\hline$I$ & The resulting intersection. \\
\hline $\mathrm{vk}_{i}, \mathrm{sk}_{i}$ & auth $_{i}$ 's public verification key and secret signing key. \\
\hline$\sigma$ or $\sigma_{i}$ & A signature issued by an authority. \\
\hline attr & An attribute attached to a signature. \\
\hline$F(x)$ & Authorities for element $x$ (for symmetric policies). \\
\hline$F\left(x, P_{i}\right)$ & Authorities for element $x$ and party $P_{i}$ (for asymmetric policies). \\
\hline$g$ & A random generator for the bilinear group. \\
\hline$R_{i}=g^{r_{i}}$ & $P_{i}$ 's challenge for the other party. \\
\hline
\end{tabular}

Policy. A privacy policy defines which authority or authorities must sign an element for a given party. For ease of exposition, we will first focus on symmetric policies, where each element needs to be authorized by one or more authorities, and the set of authorities is determined by the element itself, but is not dependent on the parties. Two exampless of symmetric policies are: (1)Claimed friendship with Alice needs to be authorized by Alice, and (2) Claimed membership in a social group needs to be authorized by the administrator(s) of the group.

As each element's authorities are determined by the element itself, we can use a function $F$ to describe symmetric policies. Formally, let $F: \mathcal{U} \rightarrow 2^{|\Lambda|}$ denote a publicly-known policy function that maps each element to the set of authorities that must sign it. For example, let $x \in \mathcal{U}$, if $F(x)=\left\{\right.$ auth $_{1}$, auth $\left._{3}\right\}$, this means that element $x$ has to be signed by authorities auth and $_{\text {auth }}$. One simple policy function is the identity function, e.g., each patient's record must be authorized by the patient herself, or claimed friendship with a user must be authorized by that user herself.

We say that $x \in \mathcal{U}$ is an authorized element for party $P$, if party $P$ has received all the necessary signatures for $x$, i.e., $P$ has received a signature $\sigma_{i}$ for every auth $_{i} \in F(x)$.

\subsection{Basic Problem Definitions}

Apart from the necessary setup and key generation functionalities, a PPSI scheme should offer two main functionalities: 1) a signature scheme allowing an authority auth $_{i}$ to authorize an element $x$ for a party $P ; 2$ ) a set intersection protocol that allows two parties to find the intersection of their authorized elements. 
We now present formal definitions for a basic PPSI scheme supporting symmetric policies. A Policy-Enhanced Private Set Intersection (PPSI) scheme (supporting symmetric policies) should provide the following algorithms or protocols:

- Setup $(\lambda)$ : The Setup algorithm is run only once at system initialization to generate public parameters param. The input $\lambda$ represents the security parameter.

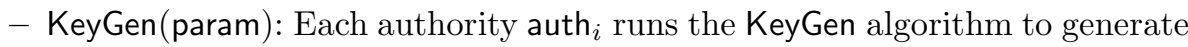
a signing and verification key pair $\left(\mathrm{sk}_{i}, \mathrm{vk}_{i}\right)$. auth $\mathrm{u}_{i}$ then announces the public verification key $\mathrm{vk}_{i}$ but keeps the private signing key $\mathrm{sk}_{i}$ to itself.

- Authorize( param $\mathrm{sk}_{i}, x, P_{j}$ ): The Authorize algorithm allows an authority auth to grant a party $P_{j}$ a signature on a specific element $x$.

- Intersect $\left(P_{i}, P_{j}, S_{i}, S_{j}\right)$ : Let $S_{i}, S_{j} \subseteq \mathcal{U}$. Intersect is an interactive protocol run by any two parties $P_{i}$ and $P_{j}$ on input sets $S_{i}$ and $S_{j}$ respectively. When both parties are honest, and assuming that $P_{i}$ and $P_{j}$ both have the necessary signatures for elements in $S_{i}$ and $S_{j}$ respectively, then both parties would learn the intersection $S_{i} \cap S_{j}$ at the end of the protocol.

\subsection{Security Definitions}

We prove the security of our protocol against a malicious adversary, who may deviate arbitrarily from the specified protocol. We define security by comparing what a malicious adversary can do in the real protocol execution against what the adversary can do in an ideal world. In the ideal-world execution, both parties would submit their sets to an imaginary trusted third-party denoted as T. The trusted party $\mathrm{T}$ would make sure that both parties have the correct authorizations on the elements they submitted. If a party submits an element without the necessary authorizations, $T$ simply ignores that element. $T$ then computes the intersection of the elements satisfying the privacy policy and returns the intersection to both parties. In the real-world, we do not use $\mathrm{T}$ and the parties communicate directly to execute the real set intersection protocol. Roughly speaking, the security definition implies that any attack that a polynomial-time adversary can perform in the real world is also possible in the ideal world. Intuitively, this suggests that the real-world set intersection protocol is as secure as the protocol in the ideal world that relies on a trusted third-party.

We now formally define the ideal functionality. The security definition involves multiple parties a subset of which is controlled by the adversary.

Authorize. $\mathrm{T}$ receives an authorization request from party $P_{i}$, requesting auth to authorize element $x$. T forwards the request to auth $_{j}$, who can either accept or

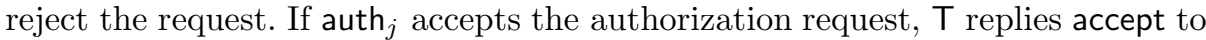
$P_{i}$ and remembers that $\mathrm{T}$ has authorized $P_{i}$ on element $x$. Otherwise, $\mathrm{T}$ replies reject to $P_{i}$.

SetIntersect. $\mathrm{T}$ receives a request from party $P_{i}$ to perform set intersection with party $P_{j}$. T forwards the request to $P_{j} . P_{i}$ and $P_{j}$ now run an ideal set intersection protocol as below (unless $P_{j}$ replies abort). 
- i) $P_{i}$ sends a set $S_{i}$ to T, and T sends $\left|S_{i}\right|$ to $P_{j}$; or ii) $P_{i}$ sends abort.

- i) $P_{j}$ sends a set $S_{j}$ to T, and T sends $\left|S_{j}\right|$ to $P_{i}$; or ii) $P_{j}$ sends abort.

- T now checks whether each element in $S_{i}$ and $S_{j}$ has appropriate authorizations. Let $\widehat{S}_{i} \subseteq S_{i}$ and $\widehat{S}_{j} \subseteq S_{j}$ denote maximal subsets of $S_{i}$ and $S_{j}$ that have appropriate authorizations. T computes the intersection $I \leftarrow \widehat{S}_{i} \cap \widehat{S}_{j}$.

- T sends $I$ to $P_{i}$, and $P_{i}$ responds ok or abort.

- T sends $I$ to $P_{j}$, and $P_{j}$ responds ok or abort.

Definition 1. Let $E=\left(E_{1}, E_{2}, \ldots, E_{m}\right)$ denote a sequence of events, where each $E_{i}$ is of the form (Authorize, $P_{i}$, auth ${ }_{j}$ ) or (SetIntersect, $\left.P_{i}, P_{j}\right)$. Let IDEAL $\operatorname{I}_{\mathcal{E}}$ denote the joint output distribution of all parties and the adversary $\mathcal{S}$ in the ideal world under event sequence $E$. Let $\operatorname{REAL}_{\mathcal{A}, E}$ denote the joint output distribution of all parties and the adversary $\mathcal{A}$ in the real world under event sequence E.

We say that a PPSI scheme is secure, if for any polynomial-time adversary $\mathcal{A}$ in the real world, there exists simulator $\mathcal{S}$ in the ideal world, such that for any sequence of events $E$,

$$
\operatorname{IDEAL}_{\mathcal{S}, E} \stackrel{c}{=} \operatorname{REAL}_{\mathcal{A}, E}
$$

where $\stackrel{c}{=}$ denotes computational indistinguishability.

Note that we cannot prevent an adversary from refusing to participate in the protocol or aborting in the middle of the protocol execution. As a result, our definition explicitly allows the ideal-world adversary to abort any time during the ideal-world protocol. Our definition also allows each party to use only a subset of their authorized elements as input to the protocol.

Our protocol is not size-hiding, i.e., each party can learn the size of the other party's set. Therefore, in the ideal functionality, the trusted third-party reveals to each party the size of the other party's set. In particular, when both parties honestly use their authorized elements as inputs, i.e., $\widehat{S}_{A}=S_{A}$ and $\widehat{S}_{B}=S_{B}$, each party learns the size of the other party's authorized set. However, notice that a party can potentially fuzz the size of its set by padding the input set with random dummy elements for which it does not possess appropriate authorizations. These dummy random elements will not appear in the final set intersection due to lack of authorizations; however, they can hide the number of authorized elements each party has.

\section{Construction}

\subsection{Strawman Schemes}

One strawman approach would be for the two parties to perform a regular Private Set Intersection (PSI) over the elements' signatures, thereby revealing the signed elements that they have in common. However, this requires that both parties have the exact same signature for the same element. This does not allow authorities to bind a signature to a specific party. The signature can thus be easily transferred to unauthorized parties. 
It is conceivable that there are other solutions based on standard techniques for the problem than our construction. For example, we can imagine schemes based on secure multi-party computation, verifiable shuffles, and matching pairs of blinded elements. However, to the best of our knowledge, these approaches all tend to have much higher computational and bandwidth complexity than our construction which achieves $O(n m+n \log \log n)$ computational overhead and $O(n)$ bandwidth overhead - both almost linear in the number of elements $n$ if we assume the number of authorities per element $m$ to be a constant.

\subsection{Preliminaries}

Bilinear group. Our scheme utilizes a bilinear $\mathbb{G}$ group of primary order $p$. There exists a non-degenerate bilinear mapping e : $\mathbb{G} \times \mathbb{G} \rightarrow \mathbb{G}_{T}$ such that $\forall g_{1}, g_{2} \in \mathbb{G}, \forall a, b \in \mathbb{Z}, \mathrm{e}\left(g_{1}^{a}, g_{2}^{b}\right)=\mathrm{e}\left(g_{1}, g_{2}\right)^{a b}$. Our scheme relies on the following computational assumption.

Computational Bilinear Diffie-Hellman (CBDH) Assumption. Let $g \in \mathbb{G}$ denote a random generator of the group. The $\mathrm{CBDH}$ assumption posits the computational hardness of the following problem. Given randomly chosen $g^{a}, g^{b}, g^{c} \in \mathbb{G}$, compute e $(g, g)^{a b c}$.

Private Set Intersection. A Private Set Intersection (PSI) protocol allows two parties to compute their set intersection without revealing other elements. Our protocol utilizes a standard PSI protocol (e.g., the scheme by Hazay and Nissim [15]) as a blackbox. We assume that the PSI protocol is secure in the malicious model, and refer the readers to [15] for a formal security definitions of PSI.

\subsection{Main Construction}

Our construction involves running a standard PSI protocol over special encodings formed as part of a challenge-response protocol. Below, we first describe our construction, including the key generation and authorization algorithm, as well as the intersection protocol. Then, in Section 3.4 we explain in detail how to construct the encodings used in the set intersection protocol, and the properties required for the encodings.

Setup. The Setup algorithm chooses a bilinear group $\mathbb{G}$ of prime order $p$ with pairing function e $: \mathbb{G} \times \mathbb{G} \rightarrow \mathbb{G}_{T}$. It then chooses a random generator $g \in \mathbb{G}$. Next, it picks a hash function $H:\{0,1\}^{*} \rightarrow \mathbb{G}$ which will be modeled as a random oracle. Finally, the Setup algorithm publishes a description of the bilinear group, the generator $g$, as well as the hash function.

Key generation algorithm. To pick a signing and verification key pair, each

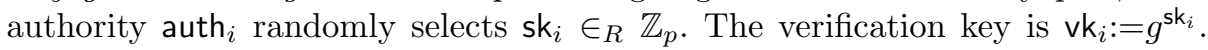
Each authority $i$ publishes its public verification key $\mathrm{vk}_{i}$, but withholds its secret signing key $\mathrm{sk}_{i}$. 
Inputs: $P_{A}, P_{B}$ each has sets $S_{A}$ and $S_{B}$, with the appropriate authorizations. Outputs: $P_{A}, P_{B}$ each obtains $I:=S_{A} \cap S_{B}$

\section{Protocol:}

1. $P_{A}: \quad$ Select random $r_{A} \in R \mathbb{Z}_{p}$, let $R_{A} \leftarrow g^{r_{A}}$

$P_{B}: \quad$ Select random $r_{B} \in_{R} \mathbb{Z}_{p}$, let $R_{B} \leftarrow g^{r_{B}}$

$P_{A} \rightarrow P_{B}: R_{A}$

$P_{B} \rightarrow P_{A}: R_{B}$

2. $P_{A}: \quad C_{A} \leftarrow\left\{\right.$ EncodeElem $\left.\left(x, r_{A}, R_{B}, P_{A}, P_{B}\right) \mid x \in S_{A}\right\}$

$P_{B}: \quad C_{B} \leftarrow\left\{\right.$ EncodeElem $\left.\left(x, r_{B}, R_{A}, P_{B}, P_{A}\right) \mid x \in S_{B}\right\}$

3. $P_{A} \Leftrightarrow P_{B}$ : Engage in a PSI protocol with input sets $C_{A}$ and $C_{B}$ respectively. As a result, both parties obtain the set $C^{\prime}:=C_{A} \cap C_{B}$ of encodings.

4. $P_{A}, P_{B}$ : Recover the intersection $I$ from their encodings $C^{\prime}$.

Fig. 1. Intersection protocol

Authorization algorithm. Let $H:\{0,1\}^{*} \rightarrow \mathbb{G}$ denote a hash function modeled as a random oracle. We assume that each party $P_{i}$ has a publicly-known unique name (e.g., an assigned name or a randomly generated identifier). Without risk of ambiguity, we overload the notation $P_{i}$ to denote either the party or its name.

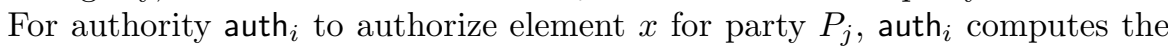
following BLS signature [5] and issues it to $P_{j}$.

$$
\sigma \leftarrow H\left(x, P_{j}\right)^{\text {sk }_{i}}
$$

In the above, the hash function is computed over the name of the element concatenated with the name of the receiving party. Notice that since the name of the party is incorporated into the signature, the signature cannot be transferred to another party.

Intersection protocol. Our protocol takes place in following four phases. The detailed construction is presented in Figure 1.

1. Challenge Phase. $P_{A}$ sends $P_{B}$ a random challenge $R_{A}$, and $P_{B}$ sends $P_{A}$ a random challenge $R_{B}$.

2. Encoding Phase. Each party computes an encoding for each element it possesses and with the appropriate authorizations. The encoding is dependent on the random challenges $R_{A}$ and $R_{B}$. Figure 2 specifies the encoding function.

3. Set Intersection Phase. Both parties perform a standard Private Set Intersection (PSI) protocol using their respective encodings as the inputs. For the underlying PSI scheme we use the protocol described in [15].

4. Recovery Phase. At the end of the PSI protocol, each party learns the intersection of the encodings. Through the intersection of encodings, each party recovers the original elements in the intersection. 


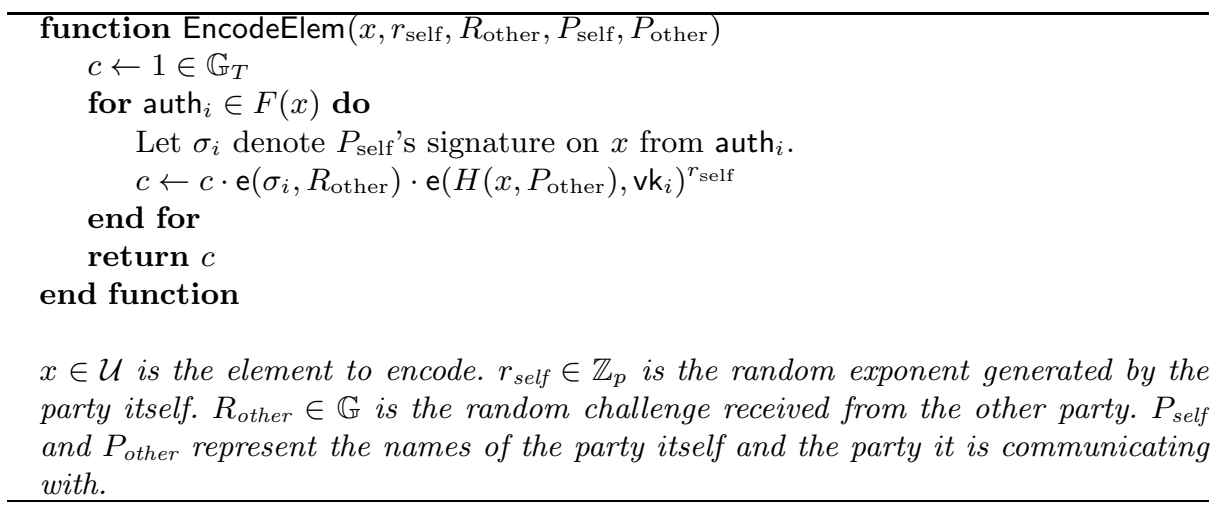

Fig. 2. The EncodeElem function

\subsection{Encodings for Symmetric Policies}

As shown in Figure 2, the encoding is computed as a product of multiple terms, where each term is the result of a bilinear pairing. Intuitively, the encodings satisfy the following properties:

- Conformity. If both parties have an element $x \in \mathcal{U}$ and the appropriate authorizations, their respective encodings of the element $x$ will be the same. Therefore, the encoding for element $x$ will appear in the intersection at the end of the PSI protocol.

- Unforgeability. If a party does not have appropriate authorizations for the element $x$, it is unable to forge the correct encoding for $x$. In this way, a dishonest party who does not possess authorizations for element $x$ cannot find out whether the other party has element $x$.

The encoding contains two corresponding terms for each element-authority pair $\left(x\right.$, auth $\left._{i}\right)$ :

- Response to the other party's challenge. The first term, e $\left(\sigma_{i}, R_{\text {other }}\right)$, is a response to the other party's challenge $R_{\text {other }}$. Intuitively, if a party does not possess an authorization from auth $_{i}$, then it will not be able to generate this part of the encoding.

- Anticipated response from the other party to one's own challenge. The second term,

$\mathrm{e}\left(H\left(x, P_{\text {other }}\right), \mathrm{vk}_{i}\right)^{r_{\text {self }}}$, is the anticipated response from the other party for one's own challenge $R_{\text {self }}$. Note that a party is always able to compute the anticipated response for its own challenge, even without knowing the other party's signature, since a party knows the exponent $r_{\text {self }}$ of a challenge generated by itself. Let $\sigma_{i}^{\prime}:=H\left(x, P_{\text {other }}\right)^{\text {sk }_{i}}$ denote the signature given to $P_{\text {other }}$

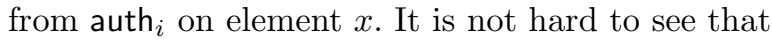

$$
\mathrm{e}\left(\sigma_{i}^{\prime}, R_{\text {self }}\right)=\mathrm{e}\left(H\left(x, P_{\text {other }}\right), \mathrm{vk}_{i}\right)^{r_{\text {self }}}
$$


In other words, if $P_{\text {other }}$ has the correct signature from auth ${ }_{i}$, its actual response to $P_{\text {self }}$ 's challenge should match the response anticipated by $P_{\text {self }}$. In summary, this term enforces that the other party can only compute the encoding if it has a signature from the correct authority.

Theorem 1. The PPSI scheme described in this section is secure against malicious adversaries, assuming 1) the underlying PSI protocol is simulatable in the malicious model; 2) the Computational Bilinear Diffie-Hellman (CBDH) assumption holds in the bilinear group $\mathbb{G}$; and 3) the hash function $H$ is a random oracle.

Proof overview. We now give an overview of our proof, and defer the detailed proof to Appendix 5. We first define a hybrid protocol by replacing the PSI protocol with an ideal functionality for PSI. Due to the sequential modular composition theorems by Canetti [7], it suffices to prove that the hybrid protocol securely computes the ideal functionality defined in Section 2 , We then construct a simulator which is given black-box access to a hybrid-world adversary $\mathcal{A}$. We show that if the encoding scheme is unforgeable in some sense, then the joint output distribution of all parties in the ideal world is indistinguishable from the joint output distribution in the hybrid world.

The description of our protocol in Figure 1 does not hide the number of authorized elements from the other party. If this number is also considered sensitive, a party can pad its set of encodings with random dummy encodings, and use the resulting set as inputs to the PSI protocol. Effectively, this reveals to the other party the total number of authorized elements and dummy elements.

Another possible method to hide the size of one's set is to use a Size-Hiding PSI protocol in place of the PSI protocol used in our construction. Our security proofs would still hold if the Size-Hiding PSI protocol is simulatable in the malicious model. Notably, Ateniese et al. recently propose a Size-Hiding PSI protocol secure under the semi-honest model [3]. Therefore, it is conceivable that a Size-Hiding PSI protocol in the malicious model will become available in the near future.

\section{Extensions for Richer Policies}

In this section, we describe how to compute the encodings in the intersection protocol for different kinds of policies. Our main construction in Section 3.3 supports simple symmetric policies, and we now incrementally add support for asymmetric policies, attributes, bundles, and DNFs.

\subsection{Asymmetric Policies}

So far we have focused on symmetric policies, where the authorities associated with each element depend on the element itself. In other application scenarios, the right authority may depend on both the element and the party performing set intersection. 


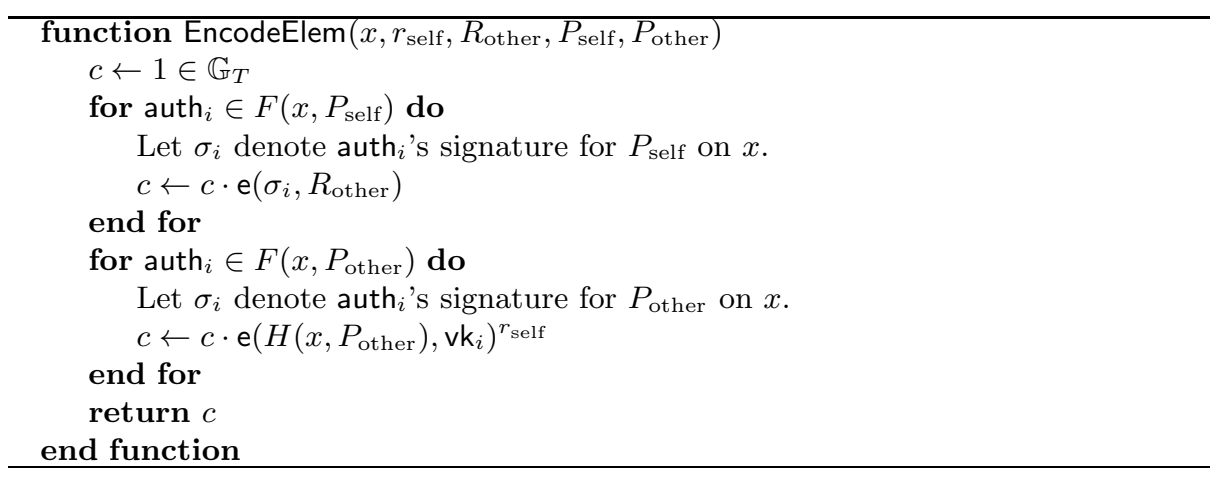

Fig. 3. The EncodeElem function for asymmetric policies

Let $\mathcal{U}$ denote a countable universe of elements, let $\mathcal{P}$ denote the set of all parties, and let $\Lambda$ denote the set of authorities. We denote asymmetric policies using a publicly known policy function $F: \mathcal{U} \times \mathcal{P} \rightarrow 2^{\Lambda}$. $F$ outputs the set of appropriate authorities given an element and a party. For example, if $F(x, P)=$

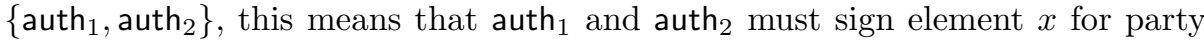
$P$.

Figure 3 describes how to modify the EncodeElem function to support asymmetric policies.

The idea is essentially the same as the symmetric case. If auth must $_{i}$ sign element $x$ for party $P_{\text {self }}$, then $P_{\text {self }}$ computes the term e $\left(\sigma_{i}, R_{\text {other }}\right)$, which is a response to the challenge from $P_{\text {other. If auth }}$ must sign element $x$ for party $P_{\text {other }}$, then $P_{\text {self }}$ computes the term e $\left(H\left(x, P_{\text {other }}\right), \mathrm{vk}_{j}\right)^{r_{\text {self }}}$, which is the anticipated response from $P_{\text {other }}$ to one's own challenge. The final encoding for an element is basically the product of all responses to the other party's challenge, and all anticipated responses from the other party.

\subsection{Attributes}

Authorities may wish to attach attributes to an element when making authorizations. For example, attributes may be used to determine the type or level of authorization given (e.g., sensitivity level of medical records). We show that our construction can be extended to support policy attributes.

Let $\mathcal{V}$ denote the set of all possible attributes. Suppose a public function $F: \mathcal{U} \times \mathcal{P} \rightarrow 2^{\Lambda \times \mathcal{V}}$ exists which outputs the necessary (authority, attribute) pairs given an element and a party. For example, if

$$
F(x, P)=\left\{\left(\text { auth }_{1}, \operatorname{attr}_{1}\right),\left(\text { auth }_{1}, \operatorname{attr}_{2}\right),\left(\text { auth }_{5}, \operatorname{attr}_{3}\right)\right\}
$$

it means that for party $P$ to be a rightful owner of element $x$, it is necessary that auth ${ }_{1}$ has signed element $x$ with attributes attr ${ }_{1}$ and $\operatorname{attr}_{2}$ for party $P$, and auth $_{5}$ has signed element $x$ with attribute $\operatorname{attr}_{3}$ for party $P$. 


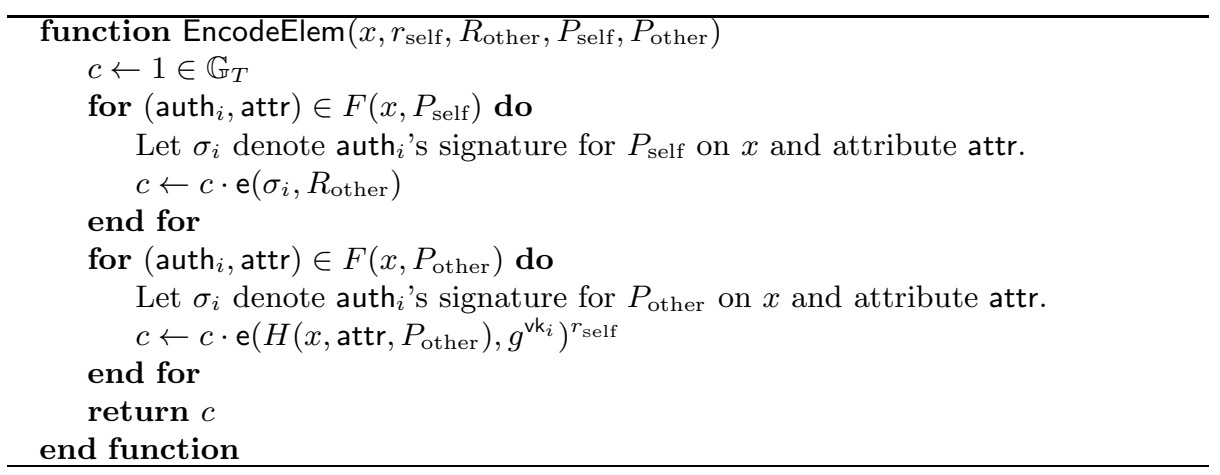

Fig. 4. The EncodeElem function supporting attributes and asymmetric policies

To support attributes, first, the authorities need to incorporate the attribute values in the hash when computing signatures. To authorize element $x$ with attribute attr to party $P$, auth $_{i}$ now computes the following signature:

$$
\sigma \leftarrow H(x, \operatorname{attr}, P)^{\text {sk }_{i}}
$$

Second, the EncodeElem function needs to be modified to incorporate the attributes as in Figure 4.

\subsection{Bundles}

A group of elements may form a bundle. The bundle should appear in the intersection if both parties have all elements in the bundle, as well as the appropriate authorizations. Otherwise, the bundle should not appear in the intersection, and neither party should learn any partial information about elements in the bundle that the other party has.

Our scheme can be easily adapted to handle bundles by combining the encoding of each element of the bundle to produce a single encoding for the entire bundle. Specifically, the bundle's encoding is the product of the encodings of its elements.

\subsection{Disjunctions and DNFs}

So far, we have considered conjunctive policies. More generally, policies may also contain disjunctions, or Disjunctive Normal Forms (DNFs). For example, if a hospital $A$ may want to share Carol's record with hospital $B$ either if the record has low sensitivity and hospital $B$ has permissions to receive low sensitivity records from Carol, or the record is cardiology related, and hospital $B$ has permissions to retrieve Carol's cardiology records.

As another example, imagine two online stores (e.g., Dell and Newegg) want to investigate a consumption pattern of their shared customers. Specifically, they 
want to determine which customers have bought both a computer from Dell and a monitor from Newegg. Therefore, they need to perform a set intersection operation on their sales datasets. Meanwhile, to prevent each company from inserting fictitious records, each sales record must be authorized by a recognized credit company, Mastercard or Visa.

In general, for parties $P_{A}$ and $P_{B}$ to share an element $x$, a DNF-style policy of the following form must be satisfied:

$$
\text { policy }:=C_{1} \vee C_{2} \vee \ldots \vee C_{k}
$$

where each $C_{i}(1 \leq i \leq k)$ is a conjunctive clause of the form:

$$
\begin{array}{r}
\left(\text { auth }_{i_{1}}, P_{A}, x, \operatorname{attr}_{1}\right) \wedge \ldots \wedge\left(\text { auth }_{i_{\ell}}, P_{A}, x, \operatorname{attr}_{\ell}\right) \\
\wedge\left(\text { auth }_{j_{1}}, P_{B}, x, \operatorname{attr}_{1}\right) \wedge \ldots \wedge\left(\text { auth }_{\ell_{\ell^{\prime}}}, P_{B}, x, \operatorname{attr}_{\ell^{\prime}}\right)
\end{array}
$$

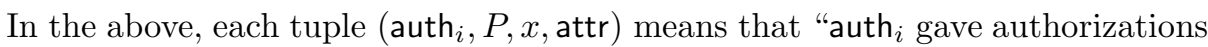
to party $P$ on element $x$ with attribute attr". Specifically, each conjunctive clause specifies the policies for party $P_{A}$ and $P_{B}$ respectively.

Our basic construction can be extended to support DNFs, with the caveat that each party reveals to the other party which conjunctive clause is satisfied for an element. The idea is quite straightforward: for each conjunctive clause, each party uses the algorithm described in Figure 4 to compute an encoding. The encoding for an element is now the union of all encodings corresponding to all conjunctive clauses. Furthermore, each party will use the union of all encodings for all elements as inputs to the PSI protocol.

\section{Proofs of Security}

Suppose the PSI protocol we use in the protocol is fully simulatable under the malicious model. Due to the sequential modular composition theorems by Canetti [7], we can replace the PSI module in our protocol with the ideal functionality for PSI. We refer to the resulting protocol as the hybrid protocol. We formally describe the hybrid protocol below. Although not explicitly stated, parties $P_{A}$ and $P_{B}$ may abort the protocol at any message boundary. The proofs for Lemma 1 and 2 are available in the full version [1] of this paper.

- $P_{A}$ picks random $r_{A} \in \mathbb{Z}_{p}$, and sends to $P_{B}$ the value $R_{A}:=g^{r_{A}} \in \mathbb{G}$.

- $P_{B}$ picks random $r_{B} \in \mathbb{Z}_{p}$, and sends to $P_{A}$ the value $R_{B}:=g^{r_{B}} \in \mathbb{G}$.

$-P_{A}$ computes $C_{A} \leftarrow$ EncodeElem $\left.\left(x, r_{A}, R_{B}, P_{A}, P_{B}\right) \mid x \in S_{A}\right\}$ and sends it to $\mathrm{T}_{\mathrm{PSI}}$. T $\mathrm{PSI}$ sends $\left|C_{A}\right|$ to $P_{B}$.

- $P_{B}$ computes $C_{B} \leftarrow\left\{\right.$ EncodeElem $\left.\left(x, r_{B}, R_{A}, P_{B}, P_{A}\right) \mid x \in S_{B}\right\}$ and sends it to $\mathrm{T}_{\mathrm{PSI}}$. T $\mathrm{PSI}$ sends $\left|C_{B}\right|$ to $P_{A}$.

- TPSI computes $C^{\prime}:=C_{A} \cap C_{B}$, and sends $C^{\prime}$ to $P_{A}$.

- T PSI sends $C^{\prime}$ to $P_{B}$.

Due to the sequential modular composition theorems by Canetti [7], it suffices to show that the hybrid protocol is secure as stated by Lemma 1 . 
Definition 2. Let $E$ denote an event sequence. Let IDEAL $_{\mathcal{S}, E}$ denote the joint output distribution of all parties and the adversary $\mathcal{S}$ in the ideal world, under event sequence $E$. Let $\operatorname{HYBRID}_{\mathcal{A}, E}$ denote the joint output distribution of all parties and the adversary $\mathcal{A}$ in the hybrid world, under event sequence $E$. We say that the hybrid protocol securely computes the ideal functionality defined in Section 2.3, if for any polynomial-time adversary $\mathcal{A}$ in the hybrid world, there exists simulator $\mathcal{S}$ in the ideal world, such that for any sequence of events $E$,

$$
\operatorname{IDEAL}_{\mathcal{S}, E} \stackrel{c}{=} \operatorname{HYBRID}_{\mathcal{A}, E}
$$

where $\stackrel{c}{=}$ denotes computational indistinguishability.

Lemma 1 (Security of the hybrid protocol). Assume that the CBDH assumption holds in the bilinear group $\mathbb{G}$, and the hash function $H$ is a random oracle. Then, the hybrid protocol described earlier securely computes the ideal functionality defined in Section 2.3.

Lemma 2 (Unforgeability of encodings). Assume that the CBDH assumption holds in the group $\mathbb{G}$, and the hash function $H$ is a random oracle. Let $\mathcal{A}$ denote polynomial-time adversary in the hybrid protocol, who has full control of all corrupted parties. Let $P_{A}$ denote a corrupted party, and assume that $P_{A}$ has not received auth $_{i}$ 's signature on element $x$. Then, during a set intersection protocol between $P_{A}$ and any honest party $P_{B}, \mathcal{A}$ is unable to compute the correct encoding EncodeElem $\left(x, r_{A}, R_{B}, P_{A}, P_{B}\right)$ except with negligible probability. In the above, $R_{B} \in_{R} \mathbb{G}$ is chosen at random by $P_{B}$, and $r_{A} \in \mathbb{Z}_{p}$ is chosen arbitrarily by the adversary $\mathcal{A}$.

\section{Performance}

In this section, we present the asymptotic complexities and experimental performance of our protocol.

\subsection{Asymptotic Complexities}

We first analyze the performance of our basic construction (described in Section 3.3) supporting symmetric policies. Later, in Section 6.3, we discuss the performance of the various extensions (described in Section 4).

The efficiency of our protocol depends on both the number of elements $(n)$ and the number of authorities per element $(m)$. We now present asymptotic bounds for the amount of computation, amount of bandwidth, and the number of communication rounds.

Computation: $O(n m+n \log \log n)$ The encoding phase performs a constant number of operations for each element-authority pair and is hence $O(n m)$. It computes a single encoding for each element resulting in $O(n)$ encodings. Those encodings are the input for the PSI phase, and by using the protocol by Hazay and Nissim [15], we can perform the PSI phase in $O(n \log \log n)$ time. The recovery phase is trivially $O(\mathrm{~nm})$ and the challenge phase is $O(1)$. Summing up the above, the total computation is $O(n m+n \log \log n)$. 
Bandwidth: $O(n)$ The communication between the two parties consists of the PSI protocol's communication and the two group elements sent during the challenge phase. Since the input size for the PSI is $n$, using the PSI protocol by Hazay and Nissim [15], the bandwidth overhead for the PSI phase is $O(n)$. Therefore, the combined communication bandwidth for our scheme is $O(n)$.

Rounds: $O(1)$ The PSI protocol by Hazay and Nissim [15] consists of $O(1)$ communication rounds. We add one additional round for the challenge phase.

Note that our construction introduces only a small overhead on top of PSI, namely, a single round of extra communication where 2 group elements are exchanged, and at most $\mathrm{nm}$ bilinear pairings. And with this small additional overhead, we provide the ability to support rich privacy policies previously not possible with existing PSI and APSI schemes.

\subsection{Empirical Performance}

Our protocol can be broken down into two time consuming phases: (1) encoding elements, and (2) performing standard Private Set Intersection (PSI). There is a large body of existing work on building efficient PSI protocols $9,10,11,12$, 13, 15, 16, 17, 18], and one can plug into our construction any existing PSI protocol that is fully simulatable under a malicious adversary model. Therefore, our experimental analysis below focuses on the additional overhead introduced by the encoding phase.

We generated 2,000 random elements with attributes and then computed the signatures for 2 parties by 5 authorities. We used different authorities for each element-authority pair, hence we have the total number of authorities $|\Lambda|=$ 10,000 . We set the maximum number of authorities per element to be $m=5$. We then varied $m=1, \ldots, 5$ by choosing a random subsets of the corresponding authorities for each element, and computed all of the the element encodings in parallel. After repeating this experiment 20 times, we calculated the average encoding time per element and standard deviation. The results are shown in Table 3 .

Our experiment was implemented in $\mathrm{C} \#$ and was run on 64 -bit Windows 7 with an Intel Core i7 3.33 GHz CPU and 12GB of RAM (although the experiment used much less memory). For all of the pairing and elliptic curve operations, we used the Pairing Based Crypto Library [19].

Table 3. The time (in ms) for encoding an element given the number of authorities for that element. These results are for the symmetric policy construction with attributes.

\begin{tabular}{|l|l|l|l|l|l|}
\hline$m$ & 1 & 2 & 3 & 4 & 5 \\
\hline \hline average & 1.70 & 3.10 & 4.45 & 5.65 & 7.07 \\
\hline std. dev. & 0.06 & 0.17 & 0.22 & 0.04 & 0.27 \\
\hline
\end{tabular}




\subsection{Performance for Rich Policies}

So far, we focused on the performance of the basic construction supporting symmetric policies. The performance of our protocols supporting richer policies can be analyzed in a similar fashion.

Asymmetric Policies. The performance for asymmetric policies is essentially the same as the performance for symmetric policies. Therefore, encoding $n$ elements each having at most $m$ authorities per element using an asymmetric policy is at least as fast as encoding using a symmetric policy for the same $n$ and $m$.

Attributes. With attribute-enriched policies, the number of bilinear pairings is the number of (element, authority, attribute) tuples for each party.

Bundle.s The cost of encoding a bundle scales linearly with the number of elements. For example, the cost of encoding a bundle of $b$ elements is $b$ times times the cost of encoding a single element. This is due to the fact that the elements of the bundle have to be first encoded individually. Combining them incurs a series of elliptic point multiplications, but their cost is significantly outweighed by the pairing function that is applied to each element.

DNF policies. Each DNF policy consists of multiple conjunctive clauses. The cost of encoding an element under a DNF policy is simply the sum of the cost of encoding each conjunctive clause, where the cost for encoding a conjunctive clause has been discussed earlier - depending on whether the conjunctive clause is symmetric, asymmetric, attribute-enriched, etc.

With a DNF policy consisting of $k$ conjunctive clauses, the encoding for an element will consist of $k$ group elements instead of one.

To summarize, suppose the maximum number of conjunctive clauses for a DNF policy is $k$, and the maximum number of literals for a conjunctive clause is $m$. Then, the communication overhead of our protocol will be $O(n k)$, and the computational overhead will be $O(n m k+n k \log \log (n k))$.

\section{Conclusion}

We introduced a new cryptographic paradigm for private set intersection with rich policies, allowing two parties to selectively share data while satisfying privacy policies. Our protocol ensures that only properly authorized elements which satisfy certain privacy policies appear in the set intersection. Our protocols support rich policies, including conjunctive and disjunctive policies, attributeenriched policies, asymmetric policies, and bundles of elements. We prove that our scheme is secure under the malicious model, given the CBDH assumption, the security of the underlying PSI protocol, and assuming the random oracle model. 


\section{References}

1. Policy-enhanced private set intersection: Sharing information while enforcing privacy policies. Techinical Report (2012), http://eprint.iacr.org/2011/509.pdf

2. Anton, A.I., Eart, J.B., Vail, M.W., Jain, N., Gheen, C.M., Frink, J.M.: HIPAA's effect on web site privacy policies. IEEE Security and Privacy 5 (January 2007)

3. Ateniese, G., Cristofaro, E.D., Tsudik, G.: Size-hiding private set intersection. Cryptology ePrint Archive, Report 2010/220 (2010), http://eprint.iacr.org/

4. Bertino, E., Ooi, B.C., Yang, Y., Deng, R.H.: Privacy and ownership preserving of outsourced medical data. In: ICDE (2005)

5. Boneh, D., Lynn, B., Shacham, H.: Short Signatures from the Weil Pairing. In: Boyd, C. (ed.) ASIACRYPT 2001. LNCS, vol. 2248, pp. 514-532. Springer, Heidelberg (2001)

6. Camenisch, J., Zaverucha, G.M.: Private Intersection of Certified Sets. In: Dingledine, R., Golle, P. (eds.) FC 2009. LNCS, vol. 5628, pp. 108-127. Springer, Heidelberg (2009)

7. Canetti, R.: Security and composition of multi-party cryptographic protocols. Journal of Cryptology (1998)

8. De Cristofaro, E., Jarecki, S., Kim, J., Tsudik, G.: Privacy-Preserving Policy-Based Information Transfer. In: Goldberg, I., Atallah, M.J. (eds.) PETS 2009. LNCS, vol. 5672, pp. 164-184. Springer, Heidelberg (2009)

9. De Cristofaro, E., Kim, J., Tsudik, G.: Linear-Complexity Private Set Intersection Protocols Secure in Malicious Model. In: Abe, M. (ed.) ASIACRYPT 2010. LNCS, vol. 6477, pp. 213-231. Springer, Heidelberg (2010)

10. De Cristofaro, E., Tsudik, G.: Practical Private Set Intersection Protocols with Linear Complexity. In: Sion, R. (ed.) FC 2010. LNCS, vol. 6052, pp. 143-159. Springer, Heidelberg (2010)

11. Dachman-Soled, D., Malkin, T., Raykova, M., Yung, M.: Efficient Robust Private Set Intersection. In: Abdalla, M., Pointcheval, D., Fouque, P.-A., Vergnaud, D. (eds.) ACNS 2009. LNCS, vol. 5536, pp. 125-142. Springer, Heidelberg (2009)

12. Freedman, M.J., Ishai, Y., Pinkas, B., Reingold, O.: Keyword Search and Oblivious Pseudorandom Functions. In: Kilian, J. (ed.) TCC 2005. LNCS, vol. 3378, pp. 303 324. Springer, Heidelberg (2005)

13. Freedman, M.J., Nissim, K., Pinkas, B.: Efficient Private Matching and Set Intersection. In: Cachin, C., Camenisch, J.L. (eds.) EUROCRYPT 2004. LNCS, vol. 3027, pp. 1-19. Springer, Heidelberg (2004)

14. Hazay, C., Lindell, Y.: Efficient Protocols for Set Intersection and Pattern Matching with Security Against Malicious and Covert Adversaries. In: Canetti, R. (ed.) TCC 2008. LNCS, vol. 4948, pp. 155-175. Springer, Heidelberg (2008)

15. Hazay, C., Nissim, K.: Efficient Set Operations in the Presence of Malicious Adversaries. In: Nguyen, P.Q., Pointcheval, D. (eds.) PKC 2010. LNCS, vol. 6056, pp. 312-331. Springer, Heidelberg (2010)

16. Jarecki, S., Liu, X.: Efficient Oblivious Pseudorandom Function with Applications to Adaptive OT and Secure Computation of Set Intersection. In: Reingold, O. (ed.) TCC 2009. LNCS, vol. 5444, pp. 577-594. Springer, Heidelberg (2009)

17. Jarecki, S., Liu, X.: Fast Secure Computation of Set Intersection. In: Garay, J.A., De Prisco, R. (eds.) SCN 2010. LNCS, vol. 6280, pp. 418-435. Springer, Heidelberg (2010)

18. Kissner, L., Song, D.: Privacy-Preserving Set Operations. In: Shoup, V. (ed.) CRYPTO 2005. LNCS, vol. 3621, pp. 241-257. Springer, Heidelberg (2005)

19. Lynn, B.: Pairing-based cryptography library, http://crypto.stanford.edu/pbc/ 